\title{
Desgaste ocupacional en docentes universitarios mediante el modelo factorial confirmatorio
}

\section{Occupational Burnout on University Teachers Through the Confirmatory Factorial Model}

\author{
Doris Fuster-Guillén* \\ Universidad Nacional Mayor de San Marcos, Lima, Perú \\ ORCID: https://orcid.org/0000-0002-7889-2243 \\ Nolan Jara-Jara \\ Universidad Nacional Mayor de San Marcos, Lima, Perú \\ ORCID: https://orcid.org/0000-0002-9467-7586

\section{Edwin Ramírez-Asís} \\ Universidad Nacional Santiago Antúnez de Mayolo, Huaraz, Perú \\ ORCID: https://orcid.org/0000-0002-9918-7607 \\ Hugo Maldonado-Leyva \\ Universidad Nacional Santiago Antúnez de Mayolo, Huaraz, Perú \\ ORCID: https://orcid.org/0000-0003-4088-6523 \\ Roger Pedro Norabuena Figueroa \\ Universidad Nacional Santiago Antúnez de Mayolo, Huaraz, Perú \\ ORCID: https://orcid.org/0000-0003-3731-9843 \\ Antonio García Guzmán \\ Universidad de Granada, Granada, España \\ ORCID: https://orcid.org/0000-0003-1495-9549
}

Recibido 12-03-19 Revisado 20-04-19 Aprobado 13-09-19 En línea 20-09-19

*Correspondencia

Email: dorisfusterguillen@gmail.com

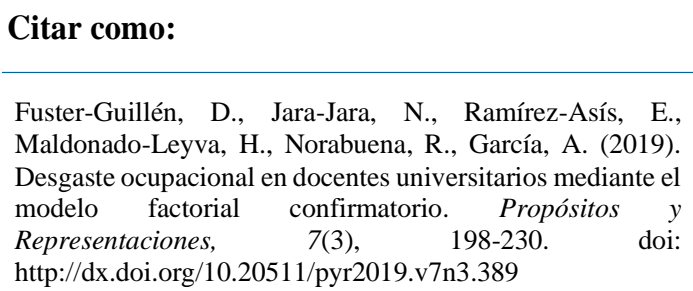

\section{Citar como}

Desgaste ocupacional en docentes universitarios mediante el

$\begin{array}{lcr}\text { Representaciones, } & 7(3), & 198-230 \\ \mathrm{http} / / / \mathrm{dx} \text {.doi.org/10.20511/pyr2019.v7n3.389 }\end{array}$ 


\section{Resumen}

La investigación aborda el desgaste ocupacional en docentes universitarios, entendida como pérdida progresiva de energía, cansancio emocional, fatiga, irritabilidad, actitudes negativas y sentimientos de insatisfacción. El propósito del estudio fue comparar la presencia de desgaste ocupacional en docentes de la Facultad de Educación e Ingeniería Electrónica de una Universidad Pública del Perú; se desarrolló según principios del enfoque cuantitativo, diseño no experimental transaccional, tipo de investigación sustantivo, descriptivo-comparativo y la muestra estaba constituida por 125 docentes de ambas facultades a los que se administró la Escala de Desgaste Ocupacional- EDO, dicho instrumento presentó validez y fiabilidad requerida. Los resultados a través del modelo factorial confirmatorio de ecuaciones estructurales, estimados mediante el método de distribución libre asintótica, permite concluir que los docentes de la Facultad de Educación presentan mayor desgaste emocional que los docentes de la facultad de ingeniería.

Palabras clave: Desgaste emocional; Agotamiento; Despersonalización; Modelo factorial.

\section{Summary}

The research addresses occupational burnout on university teachers, understood as progressive loss of energy, emotional exhaustion, fatigue, irritability, negative attitudes and feelings of dissatisfaction. The purpose of the study was to compare the presence of job burnout on teachers of the School of Education and Electronic Engineering from a Public University of Peru. It was developed according to principles of quantitative approach, transactional non-experimental design, and type of substantive, descriptive-comparative research. The sample was made up of 125 teachers from both faculties to which the Occupational Attendance Scale-EDO was administered. This instrument presented the required validity and reliability. The results obtained through the confirmatory factor model of structural equations, estimated through the asymptotic free distribution method, led to the conclusion that the teachers from the School of Education report greater emotional wear than teachers of the School of engineering.

Keywords: Emotional Exhaustion; Bunout; Depersonalization; Factorial Model.

\section{Introducción}

El desgaste ocupacional, también conocido como el síndrome del trabajador quemado (burnout) o síndrome de desgaste profesional como una forma de cansancio o desgaste originado por la dedicación a una causa, forma de vida o de relación. Freudenberger (1974), la importancia por estudiarlo ha ido en aumento en los últimos años. En una investigación llevada a cabo en más de 2.000 trabajadores de sector público y privado, se observó que el $41 \%$ presentan altos niveles de estrés laboral; dos de cada tres refirieron sentir sobrecarga de trabajo, y un 36\% manifestaron que no reciben apoyo de compañeros y supervisores. Además, uno de cada cinco no informaba sobre su alto nivel de estrés y un $48 \%$ evitaba descanso por enfermedad, ambos por miedo al despido (Paton, 2011). Un porcentaje que también se extrapola a la prevalencia del burnout en los docentes (El Sahili, 2015). Y es que, si bien este síndrome ha estado más asociado a los docentes de secundaria (León, León y cantero, 2013), también se presentan en los docentes universitarios, esto por la despreocupación del Estado Peruano en brindar condiciones laborales apropiadas a los profesores de educación superior asimismo debido a la gran cantidad de profesores pertenecientes a distintas áreas de especialización que re quieren el desarrollo de las competencias actualizadas, si a esto se suma la frecuente rotación docente y la gran cantidad de trabajo administrativo que deben realizar, además de su labor de enseñanza, entonces podrían estar más propensos a padecer burnout( Rodríguez \& Sánchez, 2018). 
El desgaste ocupacional se caracteriza por una serie de aspectos, conductas, comportamientos y condiciones organizacionales (Chavarría, Colunga, Loria \& Peláez, 2017) en las que se encuentran los docentes universitarios y que puede conllevar molestias corporales, mal humor, desgaste emocional y físico, cansancio, poca motivación, bajo rendimiento e irritabilidad. Nos referimos al debilitamiento general del sujeto, sumado a la despersonalización de las tareas que implica actitudes de rechazo (Rodríguez \& Sánchez, 2018) o el mal trato. Todo ello tiene consecuencias importantes para los principales destinatarios de nuestra enseñanza, el alumnado (Küster \& Vila, 2012), ya que se les ofrece un mal servicio, sin prestarles interés, deficiente apoyo con las tareas asignadas o no se realiza la retroalimentación adecuada ni oportuna. Todos estos aspectos se pueden entender también como síntomas psicosomáticos que trascienden, si no es identificada y atendida oportunamente.

Es importante tener en cuenta que el desgaste ocupacional afecta y se presenta en algunos docentes de la Facultad de Educación e Ingeniería electrónica tanto en el bienestar físico y mental, como en las relaciones laborales con sus compañeros (Cabezas, 2016) refiere que el docente de la facultad de Ingeniería cuenta en sus distributivos de trabajo con una carga que fluctúa de 40, 20, 12, siendo el burnout una de las consecuencias por el exceso de trabajo y condiciones sociodemográficas. Algunos docentes que trabajan en la Facultad de Educación están expuestos, son vulnerables y susceptibles de padecer agudos niveles de desgaste ocupacional, debido al gran trabajo físico, mental y emocional que genera pensar en la situación de la educación, en las personas que la conforman, las necesidades, las demandas y lo que implica enfocar y puntualizar para la atención a los estudiantes universitarios, haciendo que su labor sea rigurosa, tensa y desgastante. A todo ello es preciso sumarle la atención a situaciones de conflicto y tensión con algunos estudiantes y compañeros de trabajo, unido a ello las tareas de investigación que aún se encuentran en proceso de construcción (Arquero \& Donoso, 2013), mientras que los docentes de ingeniería no presentan acercamiento e interés en aspectos relacionados a los estudiantes, más bien se preocupan por aspectos laborales externos y no propiamente en la docencia, obvio que también conducen a despaste ocupacional.

De aquí la necesidad de estudiar el síndrome de desgaste ocupacional y las repercusiones significativas que tiene sobre la salud de la persona y la organización generando conflictos o desequilibrios en el trabajo y la familia (Patlán, 2013; Esteras, Chorot \& Sandín, 2014) (ausentismo, disminución de la productividad, disminución de la calidad, entre otros). Asimismo, cabe destacar que la mayor cantidad de casos del síndrome de desgaste laboral se dan en profesionales que prestan un servicio con una función social, según antes mencionado, desarrollándose así en un problema de salud pública. A causa de estas situaciones, resulta importante comenzar en el país investigaciones que aborden este campo de estudio con el objetivo de evaluar esta situación y de ser necesario recomendar al rectorado que adopte medidas para el cuidado de la salud ocupacional de su personal docente que amortigüe la presencia del desgaste profesional (Gaxiola-Villa, 2014), sobre todo en las facultades de ciencias sociales y humanidades. Resultado que también es consistente en otros estudios internacionales que han analizado el factor de desmotivación y siendo este mayor en el ámbito social (Pascual y Conejero, 2015). Sin embargo, en otros estudios, fueron los profesores de ciencias los que mostraron mayor “agotamiento emocional” y, por tanto, los más propensos a este síndrome (Chávez, 2017).

El desgaste ocupacional con las características ya mencionadas se presenta en algunos de los docentes de la facultad de Educación e Ingeniería. Así, en el estudio podemos observar que los más afectados son los docentes que enseñan en la Facultad de Educación, ya que dichos docentes están en estrecha relación con estudiantes que presentan intereses y necesidades diversas, además cumplen un rol de intervención, inclusión y atención a alumnos de la Educación Básica Regular en sus tres niveles; inicial, primaria y secundaria; incorporados en la pobre realidad social y mostrando múltiples carencias que deberán ser atendidas por los docentes de 
aula. Nos referimos a los estudiantes, futuros maestros, y en conjunto a quien corresponde atender todo lo manifestado es al docente universitario, que tiene un gran reto: la formación integral del futuro maestro cuya responsabilidad es la transformación de la persona en la sociedad y también preocupado por brindar una educación de calidad. Consciente de la poca práctica de valores en el país, los maestros son los llamados a insertar en el fututo docente valores como la ética, la responsabilidad, honestidad y la formación de mejores personas, lo que a la larga podría generarles desgaste y déficits en su calidad de vida repercutiendo en su salud como trastornos del sueño, ansiedad, depresión y dolor (Fernández, 2017). En los docentes de ingeniería podemos encontrar desgaste ocupacional centrados estrictamente a la presión laboral, relaciones con los compañeros de trabajo, con los estudiantes quienes presentan distintas demandas tecnológicas como diseño y creación de nuevas propuestas o prototipos electrónicos que el docente universitario no las usa porque la mayoría de ellos son de avanzada edad y se rehúsan al cambio, sus actividades de trabajo han dejado de parecer importante. Hay que destacar finalmente que aquellos que presentan mayor desgaste ocupacional son los docentes de la Facultad de Educación a los cuales se ven reflejados en el bajo desempeño laboral a medida que pasan los años. La presencia del síndrome de Burnout en docentes universitarios puede considerarse como un deterioro de la salud mental, teniendo consecuencias negativas en su desempeño laboral (García, Arlington, Escorcia \& Pérez, 2017)

A continuación, presentamos los fundamentos teóricos del desgaste ocupacional.

\section{Enfoque teórico del desgaste ocupacional}

A partir de la identificación del cansancio emocional, despersonalización, insatisfacción, así como los múltiples estudios sobre el desgaste ocupacional o síndrome de burnout han surgido diversas propuestas que se explican en diferentes modelos relacionadas a los componentes implicados. En este contexto se plantean dos perspectivas teóricas clínica y psicosocial que se hace necesario conocerlas para poder atenderlas adecuadamente.

Perspectiva Clínica. Es la perspectiva que adopta Freudenberguer (1974), quien considera el síndrome de burnout como un estado, producto de un fenómeno intrapsíquico procedente de una predisposición de la personalidad y también por el estrés en el trabajo. Desde esta mirada, la aparición del desgaste ocupacionales el resultado o consecuencia de la entrega absoluta a las labores, la utilización del trabajo como reemplazo de la vida social de trabajadores y profesionales desmesurada mente laboriosa, quienes se sienten indispensables, y que, por lo anterior, son predispuestos a presentar el síndrome. Las personas evalúan su autoestima y su autoaceptación en función de cuán importante se sienten en su centro de labores. En este sentido, ante situaciones estresantes, donde no son capaces de responder a las demandas que exige el trabajo, se presentaría el síndrome Burnout (Burisch, 2002). Los docentes universitarios, en específico los educadores por formación, siempre están involucrados con su quehacer diario, pensado en los estudiantes. No obstante después de tanto esfuerzo sienten que los objetivos planificados no se alcanzaron por situaciones que ocurren dentro del aula o institución, que provoca que muchas veces se sientan frustrados, conduciendo al estrés y, por tanto, a adquirir el síndrome de Burnout y los ingenieros encargados de impartir saberes y aprendizajes presentan incomodidades e insatisfacción personal porque consideran que fueron reemplazados y no tomados en cuenta a pesar de la dedicación y entrega por muchos años a la carrera. El desgaste ocupacional se manifiesta con cambios psicológicos que incrementan su intensidad desde el descontento y la irritabilidad hasta estallidos emocionales, en especial en profesionales en relación de dependencia, fundamentalmente en el área de prestación de servicios como es el docente universitario (Chávez, 2016).

Perspectiva Psicosocial. Desde esta mirada, el síndrome de burnout es una causa del estrés laboral crónico que se caracteriza porque el profesional se siente fracasado y muy agotado y a esto se suma las actitudes pesimistas hacia sus compañeros de trabajo. Desde esta perspectiva el síndrome de burnout es considerado como un proceso que se desarrolla a partir de la relación entre las características del entorno laboral y personal, dando lugar a diferentes manifestaciones 
en cada etapa del desarrollo del síndrome (Gil-Monte \& Peiró, 1999).En el estudio realizado, específicamente en la Facultad de Educación, se observa que los docentes se sienten no valorados, poco reconocidos por la sociedad, asimismo el clima laboral negativo entre autoridades, colegas y personal administrativo conducen al estrés docente, cansancio laboral y desmotivación que trae repercusiones personales y laborales en perjuicio a los estudiantes. La ausencia de armonía tendrá una repercusión dañina en su contexto social, familiar, personal y laboral y por dichas razones provoca trastornos emocionales y alteraciones en el conducta de los docentes universitarios (Rodríguez, Guevara \& Viramontes, 2017); al igual que en la facultad de Ingeniería Eléctrica esta perspectiva se muestra al observar el egoísmo entre colegas, envidia a quienes asumen cargos administrativos, obstaculizando los procesos de la institución, al vivenciar ello la mayoría de docentes muestra su incomodidad y rechazo produciendo estrés. En la actualidad se ha presentado un número considerable de docentes universitarios que muestran rechazo hacia la institución en la que trabajan por diversos motivos, entre ellas condiciones laborales que trae como consecuencia la aparición de estrés, crisis psicológicas y falta de motivación, problema que repercute directamente en la calidad del servicio educativo, menor interés por los estudiantes, ausentismo en las actividades propias de la institución y como punto relevante acciones negativas con respecto a sus compañeros de trabajo (Rodríguez, Guevara \& Viramontes, 2017).

La investigación asume como los modelos psicosociales, Gil-Monte y Peiró (1999, pp. 144145), las que respaldan los resultados alcanzados en la investigación estas son: teoría socio cognitiva del yo, teoría del intercambio social y teoría organizacional que a continuación se detallan.

Teoría socio cognitiva del yo. Esta teoría considera que las cogniciones de los docentes influyen en lo que éstos perciben y hacen; estas cogniciones se ven modificadas por los efectos de sus acciones y por acumulación de las consecuencias observadas en los demás y la creencia, por parte de un docente, en sus propias capacidades, lo que determinará el empeño que el sujeto pondrá para conseguir sus objetivos y la facilidad o dificultad en conseguirlos. También determinará ciertas reacciones emocionales, como son la depresión o el estrés, que acompañan la acción (Gil-Monte \& Peiró, 1999). En ambas facultades encontramos docentes con un alto nivel académico que en su día fueron un gran aporte para varias generaciones y lograron sus objetivos personales y profesionales, pero al pasar el tiempo se quedaron estáticos, no se actualizaron según las demandas académicas y administrativas de las instancias correspondientes. De esta forma, los mencionados docentes al querer obtener un cargo o ascenso se dan con la sorpresa de no cumplir con los requisitos establecidos y es ahí donde estos se sienten defraudados, desmotivados y, en definitiva, renegados de sus acciones. Según Ford (1992), se desarrolla un déficit motivacional, lo que favorece la aparición de expectativas negativas con relación a sus capacidades como maestros y sensación de ser poco competentes.

Autores como Harrinson (1983) mantienen que la competencia y eficacia percibida son elementos para el avance y desarrollo de este proceso. La mayoría de los docentes que empiezan a trabajar al servicio social a través de los estudiantes, están altamente motivados para ayudar a los demás y tiene un sentimiento de ayuda popular, puesto que la mayoría de los docentes de la Facultad de Educación antes de ser docentes universitarios fueron maestros de escuela. De esta forma, al estar tan involucrados en los problemas de los estudiantes surgen elementos que se convierten en barreras como la sobrecarga profesional, problemas interpersonales, ausencia de recursos, disfunciones de rol, etc., que obstaculizan el logro de los objetivos, reducen los sentimientos de auto eficacia y finalizan originando el síndrome de burnout. Chávez (2016) refiere que el burnout afecta de modo especial a aquellas profesiones cuyas tareas se concretan en una relación continuada y estrecha con personas, relación de ayuda y servicio en este caso particular el docente de educación superior al servicio de los estudiantes. Esta teoría socio cognitiva del yo determina su atención en la autoeficacia, autoconcepto, y autoconfianza como papel trascendental y que será clave para explicar el aparecimiento y el proceso del desgaste ocupacional en los docentes de educación superior. 
Teoría del intercambio social. Esta teoría plantea que el síndrome del quemado tiene su causa en las percepciones de falta de justicia o falta de ganancia que desarrollan los docentes universitarios como resultado del proceso de comparación social cuando establecen relaciones interpersonales (Gil-Monte \& Peiró, 1999). Los docentes universitarios establecen relaciones de compensación (ayuda, aprecio, gratitud, reconocimiento) con los compañeros del trabajo y con la universidad y la comunidad en general, cuando de manera continua percibe que aportan más de lo que reciben a cambio de su implicación personal y de su esfuerzo, desarrollan sentimientos de quemarse por el trabajo. Produce desgaste laboral cuando se presentan negativa dinámica de trabajo, relaciones tensas, competitivas, con conflictos entre compañeros y con estudiantes, falta de apoyo social, ausencia colaboración entre compañeros en tareas complementarias y ausencia de reciprocidad en los intercambios sociales (Chávez, 2016).

Esta teoría del intercambio social tiene su origen en la percepción que tiene el docente universitario respecto a la falta de igualdad, al realizar comparaciones de su labor con la de otros colegas y ven que dan más de lo que reciben creando sentimientos de desgaste laboral. Este aspecto se refleja al comparar las dos Facultades, de Educación e Ingeniería. Así, si bien el monto recibido por el trabajo realizado es proporcional entre uno u otro en la universidad, se hace necesario buscar otros ingresos que permitan cubrir las necesidades y es ahí donde se encuentra la diferencia; un docente de formación trabaja en otras instituciones educativas cuya remuneración ayuda pero no cubre lo requerido, mientras que los docentes de ingeniería no se preocupan por buscar carga lectiva, ellos realizan proyectos concursables y reciben el doble o, en algunos casos, hasta el triple de la remuneración con las que cubre todas sus necesidades. En la investigación y en el contexto se demuestra que los docentes de la Facultad de Educación trabajan más y ganan menos lo que provoca estrés, cansancio laboral y, en definitiva, se sienten quemados. El docente universitario a nivel mundial ha sufrido cambios en específico la caída de salarios, el incremento de posiciones académicas cada vez más inestables, convirtiéndolo en acciones causantes de estrés (Avargues, 2010).

Teoría organizacional. Uno de los primeros modelos de este grupo es el de Golembiewski (1983), quién destaca las disfunciones de rol (sobrecarga o pobreza de rol), como desencadenantes de las actitudes despersonalizadas que serían el elemento clave del proceso. En esta teoría organizacional, se les dedica importancia a conductas de los integrantes de un grupo u organización. Nos referimos a conductas improductivas que conducen a dispersión grupal, los conflictos y disfuncionalidad de roles entendidas como historiales del desgaste ocupacional en la organización, identificando como predictores la sobrecarga laboral, la incongruencia entre el docente universitario y su trabajo y el apoyo social en las relaciones interpersonales en el trabajo, la falta de salud organizacional, la estructura y el clima organizacional. Chávez (2016) refiere que las causantes de estrés para el docente universitario se presentan cuando no puede actuar sobre factores como el control del tiempo, la ambigüedad del rol, sobrecarga de trabajo, exigencias emocionales en la interacción con los estudiantes, falta de tiempo para la atención, falta de control de los resultados de las asignaciones, tareas inacabadas que no tienen fin, poca autonomía en las decisiones.

El docente universitario, específicamente de la Facultad de Educación, se siente quemado porque observa y vive día a día con la ineficaz labor de las autoridades, que no respetan los acuerdos, concursos, no se preocupa por atender las demandas y necesidades de los estudiantes y más bien su labor está orientada a satisfacer demandas políticas y creando así distanciamiento, inconformidad, pasividad, conflicto entre compañeros que, a su vez, provoca desgaste y agotamiento por intentar que las cosas mejoren, no se encuentra respuesta, además se denotan ciertas incongruencias como docentes observados o tachados por lo estudiantes que siguen dictando dichas cátedras perjudicando los aprendizajes. La estructura de la organización muy jerarquizada y rígida, falta de apoyo instrumental por parte de la organización, exceso de burocracia, "burocracia profesionalizada", falta de coordinación entre las unidades, relaciones conflictivas en la organización, estilo de dirección inadecuado y la forma en que se presta el servicio es relevante para identificar problemas de burnout (Chávez, 2016). Situación contraria, 
en la Facultad de Ingeniería se cumplen las normas establecidas y constantemente se realizan capacitaciones para la mejora académica de los docentes, con el objetivo de que repercuta en los estudiantes. Por lo manifestado podemos asumir que los docentes de la facultad de educación presentan altos niveles de desgaste ocupacional que a continuación definiremos.

\section{Desgaste ocupacional}

Las definiciones asumidas en la investigación Desgaste Ocupacional en docentes universitarios son la de Uribe (2010, p. 450) que cita a Freudenberger (1974), quien define el desgaste ocupacional "como un estado psicológico individual caracterizado por un conjunto de síntomas físicos y psíquicos". Este autor representó una serie de síntomas y conductas que experimentaban los profesionales que proporcionaban servicios, denominándolo en el idioma inglés como burnout, trasladada a la educación nos referimos al estado psicológico del docente que se manifiesta con molestias físicas, corporales y mentales, asimismo, Uribe (2010, p. 450) cita a Maslach y Pines (1977) quienes definieron el Desgaste Ocupacional como: Un síndrome de agotamiento físico y emocional que implica el desarrollo de actitudes negativas hacia el trabajo, pobre auto concepto, y pérdida del interés por los estudiantes que aparece en los profesionales de las organizaciones que brindan servicios educativos. En los docentes de educación superior como actitudes pesimistas a causa de tener un agotamiento físico y emocional; así como sentimiento de encontrarse al límite, de no poder dar más de si desde el punto de vista profesional y de haber agotado todos los recursos emocionales (García, Escorcia \& Pérez, 2017). Las mismas que se presentan en los docentes; como egoísmo por el crecimiento profesional de otros, desinterés por la innovación en la ciencia y tecnología que los estudiantes requieren, impotentes por mejorar y saber que no se puede hacer nada ya que los obstáculos son las autoridades. Habría que decir también que Uribe (2010, p. 450), citando a Maslach (1982), describe el desgaste ocupacional como un síndrome de contestaciones crecientes compuestas por sentimientos de extenuación emocional (desgaste emocional), actitudes negativas hacia los educandos(despersonalización), una predisposición a evaluarse así mismo de manera negativa y atañendo sentimientos de insatisfacción con el trabajo docente. También, Perlman y Hartman (1982) concluyeron que el burnout es una contestación al estrés emocional arraigado con tres elementos; agotamiento emocional y/o físico, baja productividad laboral y un exceso de despersonalización. El agotamiento y el estrés traen consigo el bajo rendimiento de los docentes frente a los estudiantes que requieren ser atendidos. De ahí que algunos estudios también con profesorado universitario destacan que "quemarse en el trabajo está significativamente relacionado con el modo de afrontamiento del estrés" (Guerrero, 2001, p.12). Además, es preciso destacar que según algunos estudios (Esteras, Chorot \& Sandín, 2014) el estrés laboral y la baja satisfacción laboral son poderosos predictores del agotamiento emocional.

\section{Factores de riesgo para el desarrollo del Síndrome de Burnout o desgaste ocupacional en docentes universitarios}

Los factores de riesgo que provocan desgaste ocupacional o desarrollo del Síndrome de Burnout en docentes universitarios se clasifican en cuatro factores de conflicto estos son: factores personales, factores de relaciones interpersonales, factores sociales y factores organizacionales (Ourcilleón, Abarca, Puentes \& Rodríguez, 2007).

Factores de riesgo Individuales. Entre los aspectos particulares para el desarrollo del síndrome de burnout se encuentran las características personales, sentimientos altruistas e idealistas, reforzados al abordar la profesión del quehacer docente, que lo llevan a implicarse excesivamente en los problemas de sus educandos (Ourcilleón, Abarca, Puentes \& Rodríguez, 2007). Esto conlleva a que se conciban culpables en los errores, tanto propios como ajenos, lo que induce una disminución de su realización profesional en el trabajo y en un aumento del agotamiento emocional. El profesional trata de distanciarse de los miembros del equipo con los que trabaja, manifiesta irritabilidad, ironía e incluso mostrando un trato despectivo, atribuyendo culpabilidad de sus frustraciones y de su descenso del desempeño (Guerrero Barona, 2003; Castro, 
2005; Tifner, 2006). Así, podemos observar en las aulas universitarias a docentes cansados, irritados, malhumorados que trasciende o se ve reflejado en la atención a los estudiantes, es decir, clases aburridas, monótonas y por ende a un malestar de los estudiantes con los docentes que provoca estrés y desgaste ocupacional en el personal docente. El desgaste ocupacional se acompaña de un incremento en la irritabilidad y una pérdida de motivación hacia el trabajo (Chávez, 2016)

Factores de riesgo de Relaciones Interpersonales. Dentro de las relaciones interpersonales para el desarrollo del síndrome de burnout, se encuentran las relaciones interpersonales tensas, complicadas y extensas con estudiantes, docentes de igual o diferente categoría como auxiliar, asociado y principalmente por la falta de soporte en el trabajo entre unos a otros, como también a los supervisores y a la propia administración de la universidad o la excesiva identificación del profesional con el educando y con las conductas y sentimientos de los colegas (Ourcilleón, Abarca, Puentes \& Rodríguez, 2007). Al encontrarnos en una institución universitaria implica que estamos en constante interacción con los estudiantes, autoridades, colegas y personal administrativo, se observa el clima organizacional laboral deteriorado por temas de poder en la Facultad de Educación existen dos grupos de docentes, uno que respalda el trabajo de la autoridad, a pesar de las malas decisiones o gestiones y otro que está preocupado por el desarrollo académico de la facultad, estos conflictos hacen que los docentes se estresen preocupándose por mejorar situaciones que políticamente no son respaldadas. Las relaciones con los colegas es una actividad diaria en muchos de los casos conducentes al burnout, provocando una serie de factores de riesgo como trato con compañeros de trabajo problemáticos, negativa dinámica de trabajo, relaciones tensas, competitivas, con conflictos entre docentes además ausencia de ayuda en los trabajos colectivos; mientras que en la facultad de ingeniería las cosas son contrarias las acciones de la autoridad permiten el crecimiento académico de la misma (Chávez, 2016).

Factores de riesgo Social. Entre los riesgos sociales para el desarrollo del síndrome de burnout se suman la aparición de nuevas leyes y estatutos que regulan el ejercicio de la profesión, nuevas instrucciones para labores y funciones debido al rápido progreso de la tecnología, así como cambios en los programas de formación de los profesionales, en los perfiles demográficos de la población (que requiere un cambio del rol profesional) y en el aumento de la demanda en los servicios por la población (Ourcilleón, Abarca, Puentes \& Rodríguez, 2007).La docencia es una especialidad que debe estar en constante cambio, en las aulas universitarias tenemos estudiantes que solicitan que sus maestros estén actualizados, que manejen tecnologías de información y comunicación. Sin embargo, aún las aulas de la Facultad de educación e ingeniería encontramos algunos docentes tradicionalistas que no se actualizan y que tienen rechazo al uso de tecnología de la información y comunicación es así como los docentes, en la búsqueda de nuevas herramientas, se agotan porque mucho de ellos no quieren salir de su zona de confort conduciendo al estrés y malestar docente. El desarrollo de las funciones como docente universitario permite identificar problemas burnout, en este caso la estructura de la organización muy jerarquizada y rígida, la ausencia de la formación práctica a los trabajadores en nuevas tecnologías y estrategias para atender a las nuevas generaciones ocasiona desgaste en algunos de los profesionales (Chávez, 2016)

Factores de riesgo Organizacional. Entre los riesgos organizacionales, se debe tener en cuenta que la profesión se desarrolla en estructuras con un esquema burocrático profesionalizado, que deriva complicaciones de coordinación entre los miembros, de libertad de acción, incapacidad de los profesionales, agregación de innovaciones, y respuestas disfuncionales, por parte de la dirección a los problemas organizacionales (Ourcilleón, Abarca, Puentes \& Rodríguez, 2007). En la universidad se observan "controles políticos" que no permiten el avance académico, si bien hay docentes preocupados en la formación integral de los estudiantes, hay otros preocupados por obtener el poder para intereses económicos y políticos, olvidando el rol fundamental del docente universitario, es decir, los estudiantes quienes son la razón de la existencia de la universidad. Asimismo, por estos "manejos" se dejan de atender a los laboratorios, en las aulas no se tienen 
equipos de última generación y los talleres en las especialidades de inicial, primaria, biología, química y física están desfasados, ya que los presupuestos han sido destinados a beneficios de quienes tiene el poder. La organización de la universidad presenta factores prevalentes de burnout como la estructura jerarquizada y rígida, exceso de burocracia, "burocracia profesionalizada", falta desarrollo profesional y estilo de dirección inadecuado (Chávez, 2016)

\section{Componentes del desgaste ocupacional}

Para trabajar los componentes del Desgaste Ocupacional nos referimos a Uribe (2010) que cita a Maslach y Jackson (1982), indicando que el desgaste ocupacional es un síndrome de contestaciones crecientes, formadas por sentimientos de extenuación emocional, actitudes negativas hacia los receptores de un servicio (despersonalización), una predisposición a evaluarse a sí mismo de manera negativa y relacionando sentimientos de insatisfacción con el puesto de trabajo. En su Escala de Desgaste Ocupacional (EDO) propone cuatro elementos: agotamiento, despersonalización, insatisfacción de logro y psicosomáticas; las mismas que se toman en el presente estudio.

Componente agotamiento. Uribe (2010) citando a Maslach y Jackson (1982), se refiere al agotamiento, como a la pérdida paulatina de energía, cansancio, desgaste y fatiga, los trabajadores sienten que ya no pueden dar más de sí mismos a nivel afectivo. Al mismo tiempo Chavarría, Colunga, Loria y Peláez (2017) refieren que el agotamiento es la sensación de fracaso como resultado de las exigencias de energías y fuerza espiritual del trabajador, presentado como una situación de extenuación de la energía o los recursos emocionales propios. Una experiencia de estar emocionalmente débil debido a la relación cotidiana mantenida con personas a las que hay que comunicarse como objeto de trabajo (estudiantes, docentes y administrativos). Esta dimensión hace referencia esencialmente a los siguientes indicadores: detrimento progresivo de energía, debilidad emocional, desgaste y fatiga. Cabe destacar, igualmente, que esta condición de estar emocionalmente agotado puede observarse cuando los docentes se tornan más susceptibles, pierden la capacidad de disfrutar por las tareas que realizan y se quejan por tener mucho trabajo. Esto es debido al contacto frecuente con los educandos vinculados a la facultad de educación, a diferencia de los estudiantes de ingeniería que son atendidos por docentes que no tienen muchas preocupaciones de carácter social.

Este componente es de especial relevancia, si tenemos en cuenta que en algunos estudios (Borges, Ruiz, Rangel \& González, 2012), se pone de manifiesto cómo el agotamiento emocional ha resultado ser el área de mayor afectación en la mayoría de los docentes.

Componente 2. Despersonalización. Uribe (2010) cita a Maslach y Jackson (1982) para referirse a la despersonalización que se presenta por irritabilidad, actitudes poco asertivas y respuestas frías e interpersonales hacia las personas (estudiantes, colegas y personal administrativo, etc.). Puede considerarse como el desarrollo de sentimientos pesimistas y de conductas y sentimientos de falsedad hacia las personas del centro de labores. Estas personas son vistas por los profesionales como insensibles debido a un endurecimiento afectivo, lo que conlleva a que les culpen de sus problemas. Por otro lado, en lo que corresponde a la despersonalización, las variables a tener en cuenta serían la irritabilidad y actitudes coléricas hacia sus educandos, expresando respuestas frías e indiferentes. El cansancio provoca la despersonalización del docente, manifestándose en sentimientos negativos como falta de valoración, actitudes erradas del trabajo, bajo auto concepto, cinismo y desinterés en la atención a estudiantes (Patlán, 2013).

Componente 3. Insatisfacción de logro. Uribe, (2010) cita a Maslach y Jackson (1982), quienes mencionan que la insatisfacción de logro son respuestas negativas hacia sí mismo y el trabajo. Es la inclinación de los docentes a autoevaluarse negativamente y, de manera especial, esta autoevaluación negativa afecta la destreza en la realización de la labor docente y la relación con las estudiantes a los que forma. Las variables que se toman en cuenta en este estudio son impresiones de insatisfacción con el trabajo y escaso auto concepto en los docentes de facultad 
de educación, en comparación a los de ingeniería debido a la asignación de cargos en las áreas correspondientes, sin tener en cuenta la meritocracia. También la diferencia salarial y el reconocimiento social que tienen los docentes de formación a pesar de los esfuerzos desplegados y la sobrecarga en atención a los estudiantes. Como refiere Patlán (2013),la sobrecarga pone en riesgo al docente en la satisfacción a sus necesidades y expectativas laborales. Sobre la mirada psicológica, la sobrecarga está asociada a la insatisfacción laboral, la tensión y la baja opinión de sí mismo.

\section{Método}

El estudio corresponde al paradigma positivista siguiendo los parámetros establecidos por el enfoque de investigación cuantitativo, método hipotético deductivo, tipo sustantivo, diseño no experimental, corte transversal, descriptivo comparativo con dos grupos de estudio que pretende identificar las diferencias de componentes del desgaste ocupacional en docentes universitarios de dos facultades.

La muestra de estudio ha estado constituida por 125 docentes, 55 docentes de la Facultad de Educación y 70 de la Facultad de Ingeniería Eléctrica de la Universidad Nacional Mayor de San Marcos. Para la delimitación de los profesores integrantes de la muestra se utilizó el criterio de inclusión, conformado por docentes nombrados de las categorías de auxiliar, asociado y principal, quienes presentan desgaste ocupacional y el criterio de exclusión conformada por docentes contratados a tiempo completo y tiempo parcial.

Para la recogida de información, si bien existen numerosas escalas para la medición del burnout con diferentes características y dimensiones o reactivos objeto de medición (Torres, ElSahili \& Gutiérrez, 2019), se optó por la utilización de la técnica de encuesta y el instrumento: EDO (Escala de Desgaste Ocupacional- Burnout) sustentado por Uribe, (2010), constituido por 30 reactivos y dividido en cuatro componentes, la primera denominada agotamiento, con 9 reactivos; Despersonalización con otros 9 reactivos y la Insatisfacción de logro, con 12 reactivos; todos estos ítems o reactivos serán valorados a través de rangos que van desde totalmente en desacuerdo a total acuerdo a los que se otorga valor de 1 a 6.

Los instrumentos han demostrado validez y fiabilidad en varios estudios (García, 2010; Hurtado \& Pereira, 2012; Barrios, 2014; Uribe, López, Pérez \& García, 2014). El instrumento de desgaste ocupacional, según Uribe (2010, p. 26), pasó por la validez de contenido de interjueces y de constructo. Correlacionó significativamente y de acuerdo con la literatura internacional con variables psicosomáticas, entre otras variables. En la investigación se aplicó una prueba piloto que a partir de ello se realizó la validez de constructo (Lawshe, 1975) y la fiabilidad con alfa de Cronbach (Cronbach, 1951) por tener respuestas politómicas ordinales cuyos valores fueron: Desgaste ocupacional: 0.888 , desgaste emocional: 0.871 , despersonalización: 0.878 y la insatisfacción del logro: 0.979. para la recolección de información se solicitó la autorización de los directores de departamento, también se contó con el apoyo de colaboradores de ambas facultades ya que se tornaba complicado dado que los docentes tenían múltiples horarios. De igual modo, para el análisis de los datos se utilizó el paquete estadístico IBM SPSS Amosversión 24.0, se aplicó la normalidad multivariante de Mardia (1970), respecto a la kurtosis multivariante (critical ratio o c.r.) que resultó superior al valor teórico de 1.96, por lo que se rechaza la normalidad multivariante. Por ello, el tamaño de muestra es suficiente para la estimación del modelo factorial confirmatorio pues se cuenta con variables confiables (Iacobucci, 2010). Así, la estimación se realizó mediante el método de mínimos cuadrados de libre escala, debido al incumplimiento de la normalidad multivariante de la matriz de varianzas (Sik-Yum, 2007) del desgaste ocupacional en docentes de la UNMSM, fueron estimados mediante el método de distribución libre asintótica. 


\section{Resultados}

El modelo factorial confirmatorio mediante ecuaciones estructurales del desgaste ocupacional en docentes de la UNMSM, fue estimado mediante el método de distribución libre asintótica. Se observa en la figura 1 que el modelo es moderadamente adecuado (RMR>0,05; GFI $<0,90$; AGFI $<0,90$; NFI $<0,90)$ para los docentes de la Facultad de Ingeniería, pudiendo deberse a la influencia del tamaño de muestra (Byrne, 2016) y con buen ajuste del modelo (RMR>0,05; GFI>0,90; AGFI>0,90; NFI<0,90) para los docentes de la Facultad de Educación (Ramlall, 2017).

Asimismo, se observan los coeficientes estandarizados y la estimación del coeficiente de determinación para cada ítem, donde resalta que en los docentes de la facultad de ingeniería, el ítem 5 es el de mayor coeficiente estructural con un $74 \%$ de variabilidad explicada por la dimensión desgaste emocional. El ítem 9 es el de mayor coeficiente estructural con un 59\% de variabilidad, explicada por la dimensión despersonalización y el ítem 1 es el de mayor coeficiente estructural con un $51 \%$ de variabilidad, explicada por la dimensión insatisfacción de logro. Mientras que en los docentes de la facultad de educación, el ítem 2 es el de mayor coeficiente estructural con un $81 \%$ de variabilidad explicada por la dimensión desgaste emocional, el ítem 29 es el de mayor coeficiente estructural con un $82 \%$ de variabilidad, explicada por la dimensión despersonalización y el ítem 30 es el de mayor coeficiente estructural con un $76 \%$ de variabilidad explicada por la dimensión insatisfacción de logro (Kline, 2016).

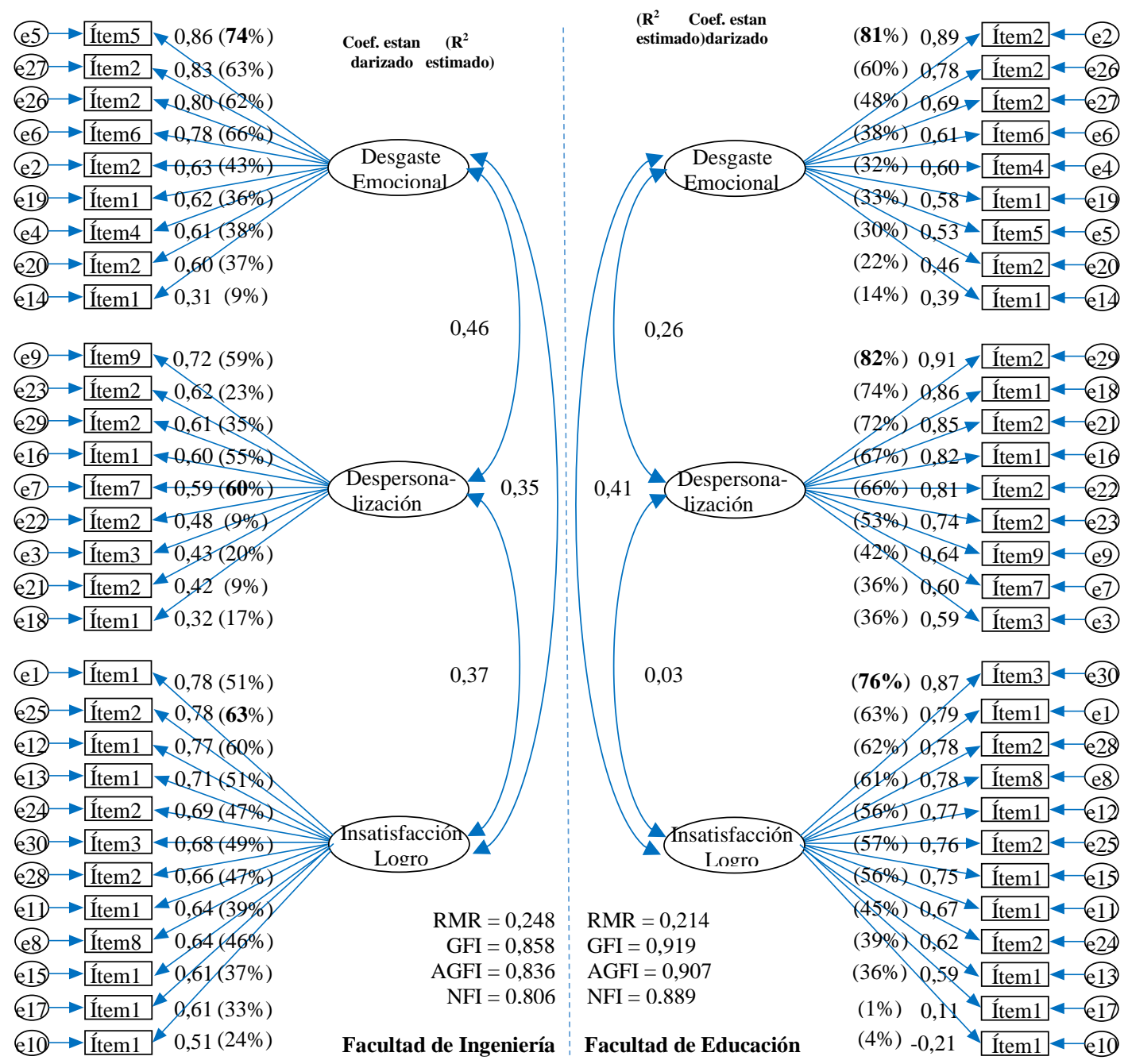

Figura 1. Modelo factorial confirmatorio mediante ecuaciones estructurales de los ítems del Desgaste Ocupacional en docentes. 

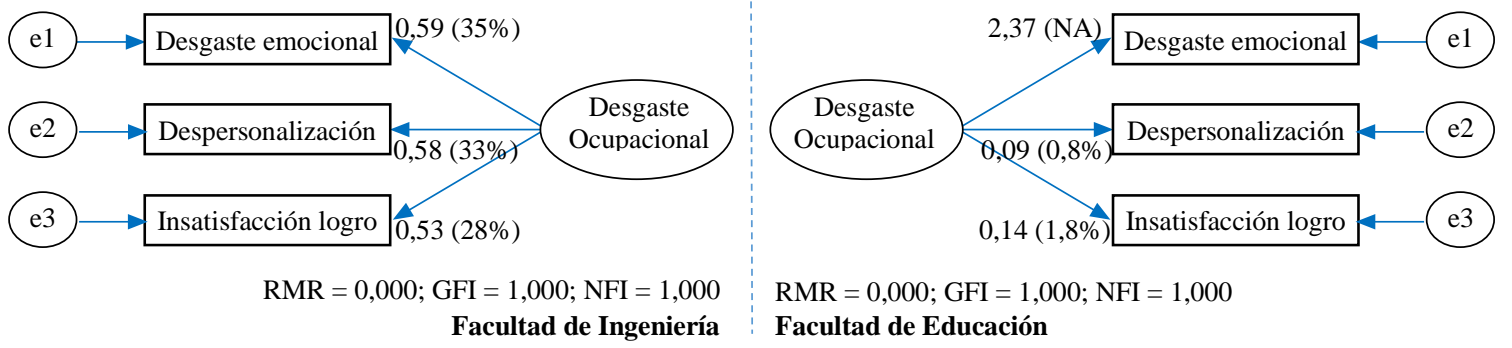

Figura 2. Modelo factorial confirmatorio mediante ecuaciones estructurales de las dimensiones del Desgaste Ocupacional en docentes.

Para ambos casos los modelos son satisfactorios (figura 2), sin embargo, resalta enormemente que en los docentes de la Facultad de Educación el desgaste emocional presenta un coeficiente estandarizado muy elevado, significando que dicha dimensión es explicada casi totalmente por el desgaste emocional, mientras que en los docentes de la facultad de ingeniería las tres dimensiones presentan coeficientes estandarizados semejantes que son explicados por el desgaste ocupacional.

\section{Discusión}

En los resultados de la investigación se muestra que los docentes de la Facultad de Educación presentan un desgaste emocional muy elevado, en comparación de otros componentes. Esto significa que en ellos está presente la pérdida progresiva de energía, debilidad emocional, desgaste y fatiga por el trabajo que demanda atender a estudiantes con munchas necesidades, que a su vez se interrelacionan con los problemas que aqueja la sociedad. Sin embargo, en los docentes de la facultad de ingeniería se presentan los tres componentes (desgaste emocional, despersonalización e insatisfacción de logro) en niveles moderados, por lo que deducimos que es gradual ya que no tienen mucho vínculo exterior $\mathrm{y}$, que, si bien están a cargo de estudiantes con múltiples necesidades, no se preocupan por los problemas socioculturales que el país demanda. En concordancia a la investigación realizada por Bareño, Berbesi y Montoya (2010) en su estudio "Factores asociados al síndrome de burnout en docentes de enfermería, Medellín-Colombia 2008", se encontró que la prevalencia de la tendencia del Síndrome de Burnout en docentes enfermeros específicamente cansancio emocional era de un $21.1 \%$. Entonces confirma que los docentes universitarios que tienen a cargo futuros docentes, que van a cumplir una labor social, corren el riesgo de desgaste ocupacional orientado específicamente al desgaste emocional propio de las funciones realizadas. Por ello es fundamental realizar acciones que la contrarresten y puedan hacer que los docentes de la facultad de educación sean más eficientes y tengan actitudes positivas.

El estudio realizado por González y Rivera (2012) encontró que según la evaluación elaborada a través de la Escala de Maslach, a los médicos residentes y agregados del Hospital Regional de Sonsonate, la sub escala con bastante nivel de afectación es el desgate emocional, al igual que la presente investigación donde se resalta que los docentes de la facultad de ingeniería, el ítem 5 (siento que un día de estos mi mente estallará de tanta presión en el trabajo)es el de mayor coeficiente estructural con un $74 \%$ de variabilidad manifestada por la dimensión desgaste emocional. Todo ello significa que lo que está causando mayor desgaste emocional es la presión que tienen los docente ingenieros en la programación curricular con un enfoque por competencias, elaboración de sílabos, preparar sesiones, estrategias enseñanza y aprendizaje, evaluación por competencias, y a esto se suma que los docentes de ingeniería tienen un pobre manejo de aspectos pedagógicos, más si los docentes son mayores de 65 años de edad; mientras que en los docentes de la facultad de educación, el ítem 2 (me cuesta mucho trabajo levantarme por las mañanas para ir a trabajar) es el de mayor coeficiente estructural, con un $81 \%$ de variabilidad explicada por la 
dimensión desgaste emocional. Esto es, debido a que sienten que tienen mucha carga académica por las tardes en el aula, atención en las tutorías, asesorías y por las noches se quedan revisando los trabajos, exámenes y programando las clases del día siguiente. De ahí que cualquier actividad programada por las mañanas tiene poca participación. Por ende, las actividades antes mencionadas provocan en el docente desgaste emocional. Asimismo, Ayala (2013) identificó el desgaste o agotamiento emocional en los trabajadores de enfermería de los servicios críticos en un nivel alto, específicamente en el cansancio al levantarse por las mañanas y tienen que empezar otro día de trabajo, además, expresan que trabajar todo el día con los pacientes implica un gran esfuerzo. Estos resultados son similares a los registrados sobre la labor de docente en la Facultad de Educación, claro está, ambas investigaciones se realizan en distintas especialidades y contextos, pero son similares por la ardua labor que ejercen y las dificultades que tienen a la hora que pretender levantarse para hacer sus actividades.

Quispe y Fuster (2016) concluyen que la dimensión de desgaste ocupacional con mayor afectación es la despersonalización, con el 57\% de los trabajadores, notándose que el nivel de afectación en las dimensiones del desgaste ocupacional son distintas en ambos estudios, por lo cual es necesario comprender las condiciones laborales en las que se desenvuelven los trabajadores, las percepciones respecto a su situación laboral y a las características individuales que tienen las personas a las que brinda el servicio o cómo perciben el trabajo que realizan y sus logros alcanzados. En concordancia con el componente despersonalización, la investigación realizada resalta que en los docentes de la facultad de ingeniería el ítem 9 (Establezco fácilmente comunicación con los usuarios de mi trabajo) es el de mayor coeficiente estructural con un 59\% de variabilidad explicada por la dimensión despersonalización, lo cual quiere decir que se realiza una atención constante a los estudiantes con diferentes problemas, necesidades que se atiende en la tutoría, el involucramiento del tutor- ingeniero con los problemas, lo que, asu vez, provoca en los docentes actitudes negativas hacia los estudiantes, aún más, cuando el estudiante no asiste a las tutorías establecidas, desarrollando en el docente la despersonalización. Mientras que, en los docentes de la facultad de educación el ítem 29 (Proporcionar un buen trato a los usuarios de mi trabajo es muy importante para mí) es el de mayor coeficiente estructural con un $82 \%$ de variabilidad explicada por la dimensión despersonalización. Así, los docentes que atienden a futuros maestros siempre se preocupan por establecer vínculos afectivos, confianza, empatía en el estudiante más aún si hablamos del enfoque por competencias que requiere el desarrollo de las habilidades cognitivas, procedimentales y con mayor énfasis las actitudinales. No obstante, algunos educandos consideran que la mejor enseñanza es aún tradicional y, por ende, no investigan, no son autónomos, claro está que ellos son producto de otros colegas que aún asumen que el aprendizaje se logra con el dominio de conocimientos del docente, impuestos mecanicamente a los estudiantes. Esta actitud, a la mayoría de los docentes de la facultad de educación, les provoca irritabilidad y emiten respuestas frías e impersonales hacia algunos estudiantes, colegas y directivos que no aceptan que la facultad debe cambiar y estar a la vanguardia en temas pedagógicos. Todo ello provoca despersonalización de los docentes que se manifiesta, a su vez, en el poco interés, actitudes negativas y por tanto bajo desempeño académico.

Los resultados de la investigación resaltan que los docentes de la facultad de ingeniería, en el ítem 1(Siento que mi trabajo es tan monótono que ya no me gusta) es el de mayor coeficiente estructural con un 51\% de variabilidad explicada por la dimensión insatisfacción de logro, manifestando que impartir los mismos conocimientos a los estudiantes año tras año resulta aburrido, lo que origina en ellos insatisfacción por el trabajo. Mientras que, en los docentes de la facultad de educación, el ítem 30 (En mi trabajo he llegado a un momento en que actúo únicamente por lo que me pagan) es el de mayor coeficiente estructural con un $76 \%$ de variabilidad, explicada por la dimensión insatisfacción de logro. Esto es debido a que los montos 
que se recibe por el trabajo realizado no retribuyen al esfuerzo y la dedicación que el docente realiza, no se reconoce ni diferencia la meritocracia dentro de la Facultad de Educación, ya que las direcciones y jefaturas en las diferentes áreas se posicionan docentes sin méritos académicos sino por manejos políticos. Todo esto provoca que los docentes estén insatisfechos por sus logros ya que no son valorados por la institución, presentándose un escaso autoconcepto que esto es ratificado por Uribe, (2010, p.3) quien refiere que, si en la institución existen actitudes y elementos perjudiciales, conducirá a evaluarse negativamente $\mathrm{y}$, de manera especial, esta evaluación negativa afecta la habilidad y competencia en la realización del trabajo y la relación con las personas que prestan el servicio. Ambas investigaciones coinciden en que lo mejor para el docente y su crecimiento profesional es tener situaciones positivas, en las que las instituciones gestionen o administren de manera correcta y meritocrática para un mejor desenvolvimiento de los docentes, con respecto a la búsqueda para ser más eficientes y competentes.

En lo que respecta a las limitaciones de este estudio, cabe destacar que no es posible generalizar los resultados, dado el tamaño de la muestra, si bien permite conocer y contrastar los resultados obtenidos por los docentes de ambas facultades e indagar sobre cómo es su desgaste ocupacional lo que, sin duda, ayudará a apostar por políticas universitarias que contribuyan a dar una respuesta adecuada a este tema. De igual modo, si bien la utilización de la escala EDO ha aportado valores adecuados de fiabilidad y validación en los aspectos evaluados y en otros estudios realizados (lo que ha determinado su utilización en este estudio), no ha sido así en otras investigaciones para determinadas variables (Torres, Al-Sahili \& Gutiérrez, 2019). Todo ello implica, a su vez, la importancia de contrastar los resultados con los obtenidos con otros instrumentos y, en definitiva, pone en evidencia la necesidad de seguir investigando este tema en el contexto universitario.

En lo que respecta a la prospectiva de investigación, el estudio realizado indica la necesidad de seguir investigando e indagando sobre este tema (desde diferentes ámbitos de conocimiento), teniendo en cuenta diferentes aspectos sociodemográficos, distintos tipos de categorías (desde asociados y ayudantes hasta catedráticos) y conjugando diferentes instrumentos y metodologías. De hecho, según algunos autores (Guerrero, 2001, p.19), "son inexistentes los estudios longitudinales y de seguimiento, ya que en general se evalúa al profesional una sola vez, en una muestra única". Y siendo conscientes de que los estudios longitudinales pueden aportar una valiosa información sobre el proceso de desarrollo y las sucesivas fases sintomatológicas del síndrome, así como las relaciones causales entre los diferentes factores personales, sociales y laborales con los que se encuentra vinculado. Finalmente, hay que destacar la importancia de indagar en las diferencias que pueden existir entre los docentes de universidades públicas y privadas. En algunos estudios se pone en evidencia las diferencias significativas en lo que respecta a condiciones de trabajo, estrés laboral y bienestar psicológico de los docentes universitarios (Milán, Calvanese \& D’Aubeterre, 2017).

Finalmente, como sugieren algunos estudios (Díaz \& Gómez, 2016; Fuster, 2019; Rodríguez \& Caurcel, 2019), es importante ampliar la investigación sobre este ámbito con otras metodologías cualitativas o mixtas para dar respuesta a las preguntas relacionadas con esta problemática a nivel regional o mundial.

\section{Referencias}

Arquero, J., \& Donoso, J. (2013). Docencia, investigación y burnout: el síndrome del quemado en profesores universitarios de Contabilidad. Revista de Contabilidad, 16(2), 94-105. doi: https://doi.org/10.1016/j.rcsar.2013.04.001

Avargues Navarro, M., \& Borda Más, M. (2010). Estrés laboral y síndrome de burnout en la Universidad: análisis descriptivo de la situación actual y revisión de las principales líneas de investigación. Anuario de Psicología Clínica y de la Salud, 6, 73-78. Recuperado de https://idus.us.es/xmlui/handle/11441/53730. 
Ayala, E. (2013). Síndrome de Burnout en el personal de enfermería de los servicios críticos del Hospital Central de la Fuerza Aérea del Perú (Tesis de especialidad). Universidad Nacional Mayor de San Marcos. Recuperado de https://pesquisa.bvsalud.org/portal/resource/pt/lil-682732?lang=es

Bareño Silva, J., Berbesi Fernández, D., \& Montoya Zapata, C. (2010). Factores asociados al síndrome de burnout en docentes de enfermería, Medellín-Colombia 2008. Investigaciones Andina, 12(21), 36-48. doi: https://doi.org/10.33132/01248146.246

Bedoya, E., Vega, N., Severiche, C., \& Meza, M. (2017). Síndrome de Quemado (Burnout) en Docentes Universitarios: El Caso de un Centro de Estudios del Caribe Colombiano. Formación Universitaria, 10(6), 51-58. doi: http://dx.doi.org/10.4067/S071850062017000600006

Burisch, M. (2002). A Longitudinal Study of Burnout: The Relative Importance of Dispositions and Experiences. Work \& Stress, 16(1), 1-17. doi: https://doi.org/10.1080/02678370110112506

Byrne, B. (2016). Structural Equation Modeling with Amos: Basic Concepts, Applications, and Programming. Nueva York: Routledge/ Taylor \& Francis.

Cabezas, E. (2016). Análisis del síndrome de Burnout en docentes de la Facultad de Ingeniería de la Universidad Nacional de Chimborazo y su incidencia en el desempeño laboral. Industrial Data, 19(1), 59-68. doi: https://doi.org/10.15381/idata.v19i1.12538.

Chavarría, R., Colunga, F., Loria, J., \& Peláez, K. (2017). Síndrome de burnout en médicos docentes de un hospital de 2. ${ }^{\circ}$ nivel en México. Revista Educación Médica, 18(4),254-261. doi: http://dx.doi.org/10.1016/j.edumed.2016.09.001

Chávez, C. (2017). Estrés, salud y psicopatías laborales. Síndrome de burnout en profesionales de la Educación Superior en Ecuador (Tesis doctoral). Universidad de Extremadura, Ecuador.

Chávez, C. (2016) Síndrome de Burnout en docentes universitarios. INNOVA Research Journal, 1(9), 77-95. doi: https://doi.org/10.33890/innova.v1.n9.2016.55

Cronbach, L. J. (1951). Coefficient alpha and the internal structure of tests. Psychometrika, 16(3), 297-334. doi: https://doi.org/10.1007/BF02310555

Díaz, F., \& Gómez, C. (2016). La investigación sobre el síndrome de Burnout en Latinoamérica entre 2000 y 2010. Psicología desde el Caribe, 33(1), 113-131. Recuperado de: http://rcientificas.uninorte.edu.co/index.php/psicologia/article/view/6442

Esteras, J., Chorot, P., \& Sandín, B. (2014). Predicción del burnout en los docentes: papel de los factores organizacionales, personales y sociodemográficos. Revista de Psicopatología y $\begin{array}{lllll}\text { Psicología Clínica, } & 19 & \text { (2), } & 79-92 .\end{array}$ https://doi.org/10.5944/rppc.vol.19.num.2.2014.13059

El-Sahili, G. L. (2015). Burnout. Consecuencias y soluciones. México D.F.: El Manual Moderno S.A. de C. V.

Ferrel, R. Pedraza, C., \& Rubio, B. (2010). El síndrome de quemarse en el trabajo (burnout) en $\begin{array}{llllll}\text { docentes universitarios. } & \text { Duazary, } & 7 & \text { (1), }\end{array}$ https://doi.org/10.21676/2389783X.306

Ford, M.E. (1992). Motivating humans. Goals, emotions and personal agency beliefs. Newbury Park, California, Estados Unidos: SAGE Publications.

Freudenberg, H. (1974). Staff burn-out. Journal of Social Issues, 30, 159-166. doi: https://doi.org/10.1111/j.1540-4560.1974.tb00706.x

Fuster, D. (2019). Investigación cualitativa: Método fenomenológico hermenéutico. Propósitos y Representaciones, 7(1), 201-229. doi: http://dx.doi.org/10.20511/pyr2019.v7n1.267

García, A., Escorcia, C., \& Pérez, B. (2017). Síndrome de Burnout y sentimiento de autoeficacia en profesores universitarios. Propósitos y Representaciones, 5(2), 65 - 126. doi: http://dx.doi.org/10.20511/pyr2017.v5n2.170

García, F. (2010). Síndrome de desgaste profesional en enfermeras que trabajan en el hospital regional de psiquiatría (Tesis de maestría), México, D.F.

Gaxiola-Villa, Eunice. (2014). Bienestar psicológico y desgaste profesional [DP] en amas de casa docentes universitarias. Journal of behavior, health \& social issues, 6(1), 79-88. https://doi.org/10.5460/jbhsi.v6.1.47605 
Gil Monte, P.R. (2005). El síndrome de quemarse por el trabajo (burnout). Una enfermedad laboral en la sociedad del bienestar. Madrid: Pirámide.

Gil-Monte, P.R., \& Peiró, J.M. (1999). Perspectivas teóricas y modelos interpretativos para el estudio del síndrome de quemarse por el trabajo. Anales de psicología, 15(2), 261-268. Recuperado de https://revistas.um.es/analesps/article/view/30161

González, Y., \& Rivera, K. (2012). Presencia del síndrome de agotamiento profesional entre los médicos agregados y residentes del Hospital Regional de Sonsonate. (Tesis de especialidad), Universidad de El salvador, El Salvador.

Guerrero Barona, E. (2001). Una investigación con docentes universitarios sobre el afrontamiento del estrés laboral y el síndrome del "quemado". Revista Iberoamericana de Educación, 25(1), 1-22. doi: https://doi.org/https://doi.org/10.35362/rie2512911

Harrison, W.D. (1983). A social competence model of burnout. En: Farber, B. A. (Ed.), Stress and burnout in the human services professions. Oxford: Pergamon.

Hurtado, D., \& Pereira, F. (2012). El síndrome de desgaste profesional (burnout syndrome): manifestación de la ruptura de reciprocidad laboral. Revista Salud Bosque, 2(2), 29-38. doi: https://doi.org/10.18270/rsb.v2i2.61

Iacobucci, D. (2010). Structural equations modeling: Fit Indices, sample size, and advanced topics. Journal of Consumer Psychology, 20, 90-98. Doi: https://doi.org/10.1016/j.jcps.2009.09.003.

Kline, R. (2016). Principles and Practice of Structural Equation Modeling (4ta ed.). Nueva York: The Guilford Press,

Küster, I., \& Vila, N. (2012). El docente universitario y sus efectos en el estudiante. Estudios sobre Educación, 23, 157-182. Recuperado de https://www.unav.edu/publicaciones/revistas/index.php/estudios-sobreeducacion/article/view/2055

Lawshe, C. H. (1975). A quantitative approach to content validity. Personnel Psychology, 28(4), 563-575. doi: http://dx.doi.org/10.1111/j.1744-6570.1975.tb01393.x

León, J., León, J., \& Cantero, F. (2013). Prevalencia y factores predictivos del burnout en docentes de enseñanza pública: el papel del género. Estrés y ansiedad, 19(1), 11-25. Recuperado de https://ciencia.iscte-iul.pt/public/pub/id/10079

Manassero, M. A., García, E., Vázquez, A., Ferrer, V. A., Ramis, C., \& Gili M. (2000). Análisis causal del burnout en la enseñanza. Revista de Psicología del Trabajo y de las Organizaciones, 16(2), 173-195.

Mardia K.V., Kent, J. T., \& Bibby J.M. (1979). Multivariate Analysis. Estados Unidos: Academic Press.

Marenco, A., \& Ávila, J. (2016). Burnout y problemas de salud mental en docentes: diferencias según características demográficas y sociolaborales. Psychología: Avances de la Disciplina, 10(1), 91-100. DOI: https://doi.org/10.21500/19002386.2469

Maslach, C., \& Jackson S.E. (1997). MBI-Inventario burnout de Maslach. Sindrome del quemado por estrés laboral asistencial. Publicaciones de Psicología Aplicada. Serie menor, 211. Madrid: TEA Ediciones.

Milán, A., Calvanese, N., \& D’Aubeterre, M.E. (2017). Condiciones de trabajo, estrés laboral, dependencia universitaria y bienestar psicológico en docentes universitarios. REDU. Revista de Docencia Universitaria, 15(1), 195-218. doi: https://doi.org/10.4995/redu.2017.6009

Ourcilleón, A., Abarca, C., Puentes, M.V., \& Rodríguez, P. (2007). Burnout en Profesionales de Enfermería de Cuidados Intensivos. Revista Chilena de Medicina Intensiva, 22(2), 83-92.

Pascual, A., \& Conejero, S. (2015). La desmotivación del profesorado universitario y su relación con variables sociodemográficas, laborales y de personalidad. Apuntes de psicología, 33 (1), 5-16. Recuperado de http://apuntesdepsicologia.es/index.php/revista/article/view/557

Patlán, J. (2013). Efecto del burnout y la sobrecarga en la calidad de vida en el trabajo. Estudios Gerenciales, 29, 445-455.

Paton, N. (2011). Time to tackle stress at work. Occupational Health, 63(6), 17-19.

Perlman, B., \& Hartman, E. A. (1982). Burnout: summary and future research. Human Relations, 35(4), 283-305. doi: http://dx.doi.org/10.1177/001872678203500402 
Quispe, Y., \& Fuster, D. (2016). Desgaste ocupacional en profesionales del área de tratamiento de los establecimientos penitenciarios de mujeres de Chorrillos, 2015. PsiqueMag, 5(1), 125-133. Recuperado de http://ojs.ucvlima.edu.pe/index.php/psiquemag/article/view/157

Ramlall, I. (2017). Applied structural equation modelling for researchers and practitioners: Using $\mathrm{R}$ and Stata for behavioural research. Emerald Group Publishing.

Rodríguez Flores, E., \& Sánchez, M. (2018). Síndrome de Burnout y variables sociodemográficas en docentes de una universidad privada de Lima. Revista de Investigación Educativa, 36(2), 401-419. doi: http://dx.doi.org/10.6018/rie.36.2.282661.

Rodríguez Fuentes, A., Caurcel, $M^{\mathrm{a}}$. J. (2019). Aproximación cualitativa del escudriño en Psicología educativa, Propostitos y Representaciones, 7(1), 1-9, doi: http://dx.doi.org/10.20511/pyr2019.v7n1.301.

Rodríguez Ramírez, J., Guevara Araiza, A., \& Viramontes Anaya, E. (2017). Síndrome de burnout en docentes. Revista de investigación educativa de la REDIECH, 8(14), 45-67. Recuperado de https://www.rediech.org/ojs/2017/index.php/ie_rie_rediech/article/view/39

Sik-Yum, L. (2007). Structural Equation Modeling: A Bayesian Approach. Estados Unidos: Wiley.

Torres, E.F., Al-Sahili, L.F., \& Gutiérrez, V. (2019). Análisis del burnout y sus escalas de medición. Praxis investigativa REDIE, 11(20), 106-124. Recuperado de http://praxisinvestigativa.mx/assets/praxisinv20.pdf\#page $=106$

Uribe, J., López, P., Pérez, C., \& García, A. (2014). Síndrome de Desgaste Ocupacional (Burnout) y su Relación con Salud y Riesgo Psicosocial en Funcionarios Públicos que Imparten Justicia en México, D.F. Acta de Investigación Psicológica, 4(2), 1554-1571. doi: https://doi.org/10.1016/S2007-4719(14)70393-X

Uribe, J.F. (2010). EDO Escala de Desgaste Ocupacional (Burnout). México: Editorial el Manual Moderno.

Zabalza, M. (2009). Ser profesor universitario hoy. La cuestión universitaria, 5, 68-80. Recuperado de http://polired.upm.es/index.php/lacuestionuniversitaria/article/view/3338 